\title{
5H-Oxazol-4-ones as Building Blocks for Asymmetric Synthesis of $\alpha$-Hydroxycarboxylic Acid Derivatives
}

\author{
Barry M. Trost*, Kalindi Dogra and Maurizio Franzini
}

\section{Experimental section ${ }^{1}$}

All reactions were carried out in oven-dried flasks or pyrex test tube under a positive pressure of nitrogen, argon for Mo-catalyzed AAA. Anhydrous solvents and bases were obtained either from elution through alumina (DCM), or from refluxing on a suitable drying agent (and distilled under reduced pressure where required): $\mathrm{CaH}_{2}$ for DCE, py; Na-benzophenone ketyl for THF; DMF and DMA were used directly from commercial sources (stored on $4 \mathrm{MS}$ ). THF solutions of LHMDS were freshly prepared by dissolving commercially available solid LHMDS (stored in dry box) in freshly distilled THF. Microwave assisted heating was carried out in a Smith Workstation US manufactured by Personal Chemistry AB. TLC was performed on precoated glass plates (Merck), flash chromatography with silica gel 60, 230-400 mesh, in compliance with Still s method. Detection of compounds on TLC was carried out either by visualization with a $254 \mathrm{~nm}$ lamp, or by exposure to an atmosphere of iodine (supported on sand), or by dipping into a suitable staining solution (A: $1.5 \% \mathrm{KMnO}_{4}, 10 \% \mathrm{~K}_{2} \mathrm{CO}_{3}, 2 \% \mathrm{NaOH}$ in $200 \mathrm{~mL} \mathrm{H} \mathrm{H}_{2} \mathrm{O}$; B: $0.42 \mathrm{~mL} p$-anisaldehyde, $1.5 \mathrm{~mL} \mathrm{AcOH}, 5 \mathrm{~mL} \mathrm{H} \mathrm{SO}_{4}, 135 \mathrm{~mL}$ EtOH; C:

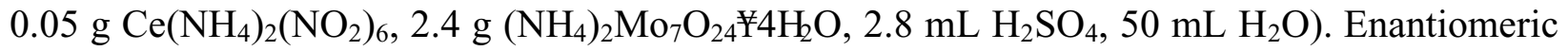
excesses were determined by HPLC on Tsp Spectraseries P100/UV100 apparatus with a chiral stationary phase (see details where applies). Melting points (uncorrected) were determined in open capillary tubes using a Thomas-Hoover apparatus. ${ }^{1} \mathrm{H}-\mathrm{NMR}$ ( $0 \mathrm{ppm}$ as internal standard) and ${ }^{13} \mathrm{C}$ - NMR (77 ppm as internal standard) spectra were recorded on Varian Gemini 2300 (300 $\mathrm{MHz}$ for $\left.{ }^{1} \mathrm{H}-\mathrm{NMR}\right)$, Mercury 400 (400 MHz for $\left.{ }^{1} \mathrm{H}-\mathrm{NMR}\right)$ and on Unity Inova $500(500 \mathrm{MHz}$ for $\left.{ }^{1} \mathrm{H}-\mathrm{NMR}\right)$. IR spectra $\left(\mathrm{cm}^{-1}\right)$ were obtained with a Perkin-Elmer FT-IR Paragon 500 spectrometer, either using neat sample on $\mathrm{NaCl}$ pad (for oils and liquids), or preparing a $\mathrm{KBr}$ pellet.

\footnotetext{
${ }^{1}$ Abbreviations used throughout the text: AAA, asymmetric allylic alkylation; DCE, 1,2-dichloroethane; DCM, dichloromethane; DMA, dimethylacetamide; DMF, dimethyl formamide; Hept, heptane; Hex, hexanes; LHMDS, lithium bis(trimethylsilyl)azide; mp, melting point; PE, pethroleum ether; py, pyridine; TLC, thin layer chromatography.
} 


\section{Representative procedures for the preparation of diamides 2.}

\section{$N$-Benzoyl-2-bromopropanamide}<smiles>CC(Br)C(=O)NC(=O)c1ccccc1</smiles>

Commercially available 2-bromopropanoyl bromide $(7.5 \mathrm{~mL}, 71 \mathrm{mmol})$ was added to a solution of benzamide $(4.50 \mathrm{~g}, 37.1 \mathrm{mmol})$ and pyridine $(6.0 \mathrm{~mL}, 74 \mathrm{mmol})$ in DCM $(40 \mathrm{~mL})$ at room temperature. A gentle reflux was set for $5 \mathrm{~h}$. After cooling down to room temperature, most of the solvent was removed by rotary evaporation. The residual slurry, obtained after removal of most of solvents, was taken up in DCM, loaded onto a column of silica gel and eluted with 8:2 to 7:3 PE/EtOAc. A white crystalline solid was obtained $(8.529 \mathrm{~g}, 33.3 \mathrm{mmol})$, along with a portion of mixed fractions, containing both the desired product and the corresponding, desired oxazolone (587 mg). Yield 90\%.

$\mathrm{R}_{f}=0.55$ (75:25 PE/EtOAc; $254 \mathrm{~nm}$, stain C). ${ }^{1} \mathrm{H}-\mathrm{NMR}\left(500 \mathrm{MHz}, \mathrm{CDCl}_{3}\right), \delta: 7.85$ (2H, d, $J=7.5 \mathrm{~Hz}), 7.61(1 \mathrm{H}, \mathrm{t}, J=7.5 \mathrm{~Hz}), 7.52(2 \mathrm{H}, \mathrm{t}, J=7.5 \mathrm{~Hz}), 5.44(1 \mathrm{H}, \mathrm{q}, J=7 \mathrm{~Hz}), 1.89(3 \mathrm{H}, \mathrm{t}, J=7$ Hz), 1.57 (1H, br s). IR (neat): 3370, 2928, 1732, 1603, 1542, 1489, 1451, 1379, 1238, and $1172 \mathrm{~cm}^{-1}$.

\section{N-Benzoyl-2-bromopropanamide}

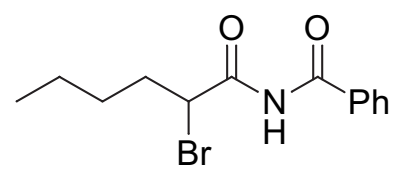

${ }^{1} \mathrm{H}-\mathrm{NMR}\left(500 \mathrm{MHz}, \mathrm{CDCl}_{3}\right), \delta: 9.00(1 \mathrm{H}, \mathrm{br} \mathrm{s}), 7.89(2 \mathrm{H}, \mathrm{dt}, J=7, J=0.2 \mathrm{~Hz}), 7.63(1 \mathrm{H}$, m), $7.53(2 \mathrm{H}, \mathrm{dt}, J=14, J=0.5 \mathrm{~Hz}), 5.37(1 \mathrm{H}, \mathrm{dd}, J=8.5, J=6.5 \mathrm{~Hz}), 2.17-2.21(1 \mathrm{H}, \mathrm{m}), 2.07-2.10$ $(1 \mathrm{H}, \mathrm{m}), 1.36-1.46(4 \mathrm{H}, \mathrm{m}), 0.94(3 \mathrm{H}, \mathrm{t}, J=7 \mathrm{~Hz}) .{ }^{13} \mathrm{C}-\mathrm{NMR}\left(125 \mathrm{MHz}, \mathrm{CDCl}_{3}\right), \delta:$ 171.0, 165.0, 133.6, 132.3, 129.1, 127.8, 48.0, 33.9, 29.4, 22.1, 13.9. 


\section{N-Benzoyl-2-bromo-3-phenylpropanamide}<smiles>O=C(NC(=O)C(Br)Cc1ccccc1)c1ccccc1</smiles>

2-Bromo-3-phenyl propanoyl chloride (380 mg, $2.75 \mathrm{mmol})$, benzamide (195 mg, 1.61 $\mathrm{mmol})$ and pyridine $(0.3 \mathrm{~mL}, 3.7 \mathrm{mmol})$ were heated in 1,2-DCE $(10 \mathrm{~mL})$ at $80{ }_{¡} \mathrm{C}$ for $1.5 \mathrm{~h}$, then at $60{ }_{\mathrm{C}} \mathrm{C}$ for an additional $15 \mathrm{~h}$. After cooling to r.t. and dilution with DCM (100 mL), the organic phase was washed with water $(50 \mathrm{~mL})$, brine $(25 \mathrm{~mL})$. After drying on $\mathrm{MgSO}_{4}$, the clear solution was concentrated, loaded onto a column of silica gel and eluted with 75:25 to $6: 4$ PE/EtOAc. The desired product was isolated as a white solid (197 mg, $0.59 \mathrm{mmol}$ ). Yield 37\%.

$\mathrm{R}_{f}=0.5\left(75: 25 \mathrm{PE} / \mathrm{EtOAc} ; 254 \mathrm{~nm}\right.$, stains A and C). ${ }^{1} \mathrm{H}-\mathrm{NMR}\left(400 \mathrm{MHz}, \mathrm{CDCl}_{3}\right), \delta: 8.84$ (1H, br s), $7.81(2 \mathrm{H}, \mathrm{d}, J=7.5 \mathrm{~Hz}), 7.60(1 \mathrm{H}, \mathrm{t}, J=6.3 \mathrm{~Hz}), 7.49(2 \mathrm{H}, \mathrm{t}, J=7.5 \mathrm{~Hz}), 7.25-7.33(5 \mathrm{H}$, m), $5.70(1 \mathrm{H}, t, J=7.2 \mathrm{~Hz}), 3.62(1 \mathrm{H}, \mathrm{dd}, J=7.5 \mathrm{~Hz}, J=14 \mathrm{~Hz}), 3.31(1 \mathrm{H}, \mathrm{dd}, J=7.2 \mathrm{~Hz}, J=14$ Hz). ${ }^{13} \mathrm{C}-\mathrm{NMR}\left(100 \mathrm{MHz}, \mathrm{CDCl}_{3}\right)$, $\delta: 170.7,165.1,136.9,133.7,129.8,129.6,129.2,128.8$, 127.9, 127.4, 47.2, 40.3. IR (neat): 3282, 3167, 3063, 3030, 1748, 1715, 1694, 1682, 1504, 1455 , $1372,1260,1145,1074$, and $695 \mathrm{~cm}^{-1}$.

\section{N-Benzoyl-2-bromo-3-methylbutanamide}<smiles>CC(C)C(Br)C(=O)NC(=O)c1ccccc1</smiles>

$\mathrm{R}_{f}=0.60$ (75:25 PE/EtOAc; $254 \mathrm{~nm}$, stains A and C). ${ }^{1} \mathrm{H}-\mathrm{NMR}\left(500 \mathrm{MHz}, \mathrm{CDCl}_{3}\right), \delta: 9.34$ $(1 \mathrm{H}$, br s), 7.85-7.89 $(2 \mathrm{H}, \mathrm{m}), 7.61-7.66(1 \mathrm{H}, \mathrm{m}), 7.50-7.55(2 \mathrm{H}, \mathrm{m}), 4.95(1 \mathrm{H}, \mathrm{d}, J=8 \mathrm{~Hz})$, 2.53-2.60 (1H, m), $1.14(3 \mathrm{H}, \mathrm{d}, J=11.5 \mathrm{~Hz}), 1.06(3 \mathrm{H}, \mathrm{d}, J=11.5 \mathrm{~Hz}) .{ }^{13} \mathrm{C}-\mathrm{NMR}(125 \mathrm{MHz}$, $\left.\mathrm{CDCl}_{3}\right), \delta: 169.0,164.1,133.7,129.2,127.9,67.0,57.3,32.3,20.2$. 


\section{$N$-Benzoyl-2-bromo-4-methylpentanamide}

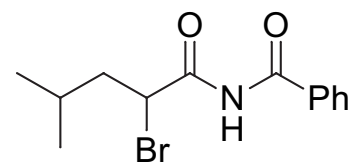

$\mathrm{R}_{f}=0.63\left(75: 25 \mathrm{PE} / \mathrm{EtOAc} ; 254 \mathrm{~nm}\right.$, stains A and C). ${ }^{1} \mathrm{H}-\mathrm{NMR}\left(400 \mathrm{MHz}, \mathrm{CDCl}_{3}\right), \delta: 9.30$ (1H, br s), 7.88-7.90 (2H, m), 7.61-7.67 (1H, m), 7.48-7.54 (2H, m), 5.22 (1H, m), 1.87-1.98 $(2 \mathrm{H}+1 \mathrm{H}, \mathrm{m}+\mathrm{m}), 1.00(6 \mathrm{H}, \mathrm{d}, J=6 \mathrm{~Hz}) .{ }^{13} \mathrm{C}-\mathrm{NMR}\left(100 \mathrm{MHz}, \mathrm{CDCl}_{3}\right), \delta: 170.7,165.1,133.7$, 132.3, 129.2, 128.0, 58.1, 43.1, 25.3, 23.0, 21.1. IR (neat): 3282, 2960, 2872, 1740, 1693, 1601, $1508,1487,1371,1263,1156$, and $707 \mathrm{~cm}^{-1}$.

\section{$N$-Benzoyl-2-bromo-2-cyclohexylacetamide}

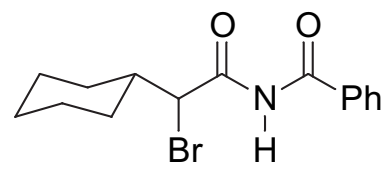

$\mathrm{R}_{f}=0.65\left(95: 5 \mathrm{CHCl}_{3} / \mathrm{MeOH} ; 254 \mathrm{~nm}\right.$, stains $\mathrm{A}$ and C). ${ }^{1} \mathrm{H}-\mathrm{NMR}\left(400 \mathrm{MHz}, \mathrm{CDCl}_{3}\right), \delta$ : $9.30(1 \mathrm{H}$, br s), $7.86(2 \mathrm{H}, \mathrm{dd}, J=1.4 \mathrm{~Hz}, J=8.2 \mathrm{~Hz}), 7.62-7.66(1 \mathrm{H}, \mathrm{m}), 7.51-7.55(2 \mathrm{H}, \mathrm{m}), 4.87$ $(1 \mathrm{H}, \mathrm{d}, J=5.2 \mathrm{~Hz}), 1.77-1.82(5 \mathrm{H}, \mathrm{m}), 1.29-1.34(5 \mathrm{H}, \mathrm{m}) .{ }^{13} \mathrm{C}-\mathrm{NMR}\left(100 \mathrm{MHz}, \mathrm{CDCl}_{3}\right), \delta$ : 168.8, 164.7, 133.7, 132.6, 129.3, 127.9, 65.9, 41.8, 30.3, 27.7, 26.0, 25.9, 25.7. IR (KBr): 3249, 2929, 2853, 1736, 1678, 1603, 1508, 1272 and $1154 \mathrm{~cm}^{-1}$. HRMS for $\mathrm{C}_{15} \mathrm{H}_{18}{ }^{79} \mathrm{BrNO}_{2}$ : found 323.05209, calc d 323.05148; for $\mathrm{C}_{15} \mathrm{H}_{18}{ }^{81} \mathrm{BrNO}_{2}$ : found 325.05004, calc d 325.05342 .

\section{$N$-Benzoyl-2-bromopent-4-enamide}<smiles>C=CCC(Br)C(=O)NC(=O)c1ccccc1</smiles>

$\mathrm{R}_{f}=0.35$ (8:2 PE/EtOAc; $254 \mathrm{~nm}$, stains A and C). ${ }^{1} \mathrm{H}-\mathrm{NMR}\left(500 \mathrm{MHz}, \mathrm{CDCl}_{3}\right), \delta: 9.27$ (1H, br s), 7.88-7.90 (2H, m), 7.62-7.65 (1H, m), 7.51-7.54 (2H, m), 5.82-5.91 (1H, m), 5.20$5.27(2+1 \mathrm{H}, \mathrm{m}), 2.90-2.96(1 \mathrm{H}, \mathrm{m}), 2.72-2.79(1 \mathrm{H}, \mathrm{m}) .{ }^{13} \mathrm{C}-\mathrm{NMR}\left(125 \mathrm{MHz}, \mathrm{CDCl}_{3}\right), \delta: 169.6$, 165.0, 133.8, 132.4, 132.1, 129.2, 128.0, 120.0, 58.3, 38.7. IR (neat): 3287, 3080, 2982, 1744, $1694,1643,1601,1505,1487,1263,1155$, and $707 \mathrm{~cm}^{-1}$. 


\section{Representative procedure for the preparation of oxalactims 3.}

5H-5-Methyl-2-phenyl-2-oxazol-4-one

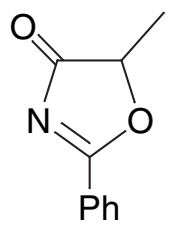

Mp: 63-65 ¡C. $\mathrm{R}_{f}=0.4$ (75:25 PE/EtOAc; $254 \mathrm{~nm}$, stain C). ${ }^{1} \mathrm{H}-\mathrm{NMR}\left(500 \mathrm{MHz}, \mathrm{CDCl}_{3}\right)$, $\delta: ~ 8.22-8.24(2 \mathrm{H}, \mathrm{m}), 7.70(1 \mathrm{H}, \mathrm{t}, J=7.5 \mathrm{~Hz}), 7.55(2 \mathrm{H}, \mathrm{t}, J=7.5 \mathrm{~Hz}), 4.85(1 \mathrm{H}, \mathrm{q}, J=7 \mathrm{~Hz}), 1.65$ $(3 \mathrm{H}, \mathrm{t}, J=7 \mathrm{~Hz}) .{ }^{13} \mathrm{C}-\mathrm{NMR}\left(125 \mathrm{MHz}, \mathrm{CDCl}_{3}\right), \delta: 192.6,186.5,135.3,130.3,129.1,127.9,125.9$, 78.2, 16.6. IR (KBr): 1758, 1605, 1589, 1549, 1490, 1451, 1368, 1276, 1186, 1030, and $710 \mathrm{~cm}^{-1}$.

\section{H-5-Butyl-2-phenyl-2-oxazol-4-one}

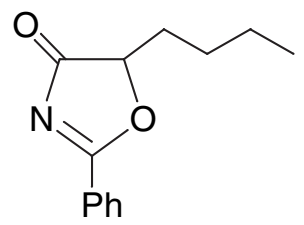

${ }^{1} \mathrm{H}-\mathrm{NMR}\left(300 \mathrm{MHz}, \mathrm{CDCl}_{3}\right), \delta: 8.22(2 \mathrm{H}, \mathrm{t}, J=7.5 \mathrm{~Hz}), 7.71(1 \mathrm{H}, \mathrm{t}, J=7.5 \mathrm{~Hz}), 7.55(2 \mathrm{H}, \mathrm{t}$, $J=7.5 \mathrm{~Hz}), 4.79(1 \mathrm{H}, \mathrm{dd}, J=7.5 \mathrm{~Hz}, J=4.5 \mathrm{~Hz}), 2.05-2.15$ (1H, m), 1.81-1.93 (1H, m), 1.34-1.54 $(4 \mathrm{H}, \mathrm{m}), 0.93(3 \mathrm{H}, \mathrm{t}, J=7.5 \mathrm{~Hz}) .{ }^{13} \mathrm{C}-\mathrm{NMR}\left(75 \mathrm{MHz}, \mathrm{CDCl}_{3}\right), \delta: 191.9,186.5,135.1,130.0$, 128.9, 127.8, 125.7, 81.9, 30.8, 26.6, 22.1, 13.7. IR (neat): 3065, 2959, 2872, 1758, 1721, 1695, $1604,1590,1552,1490,1452,1368,1242,1181,1061$, and $711 \mathrm{~cm}^{-1}$. 


\section{H-5-Phenylmethyl-2-phenyl-2-oxazol-4-one}

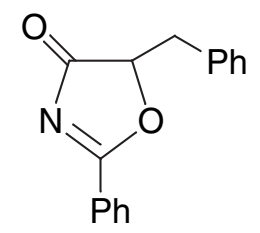

Mp: $95-98$ ¡C. $\mathrm{R}_{f}=0.35$ (75:25 PE/EtOAc; $254 \mathrm{~nm}, \mathrm{I}_{2}$, stains A and C). ${ }^{1} \mathrm{H}-\mathrm{NMR}(300$ $\left.\mathrm{MHz}, \mathrm{CDCl}_{3}\right), \delta:$ 8.14-8.16 (2H, m), 7.66-7.70 (1H, m), 7.51-7.53 (2H, m), 7.24-7.32 (5H, m), $5.00(1 \mathrm{H}, \mathrm{dd}, J=4 \mathrm{~Hz}, J=7.5 \mathrm{~Hz}), 3.44(1 \mathrm{H}, \mathrm{dd}, J=4 \mathrm{~Hz}, J=15 \mathrm{~Hz}), 3.13(1 \mathrm{H}, \mathrm{dd}, J=7.5 \mathrm{~Hz}, J$ $=15 \mathrm{~Hz}) .{ }^{13} \mathrm{C}-\mathrm{NMR}\left(75 \mathrm{MHz}, \mathrm{CDCl}_{3}\right), \delta: 191.1,186.7,135.4,134.8,130.2,129.8,129.5,129.1$, 128.8, 127.5, 125.8, 82.1, 37.4. IR (neat): 3063, 3031, 2925, 1756, 1604, 1590, 1549, 1490, 1451, 1371, 1180, 1064, and $700 \mathrm{~cm}^{-1}$. Anal. Calc d for $\mathrm{C}_{16} \mathrm{H}_{13} \mathrm{NO}_{2}: \mathrm{C}, 76.48 ; \mathrm{H}, 5.21 ; \mathrm{N}, 5.57$. Found: C, 76.24; H, 5.09; N, 5.34.

\section{H-5-(Methyl)ethyl-2-phenyl-2-oxazol-4-one.}

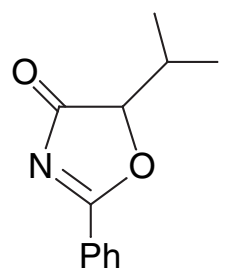

Mp: 79-81 ¡C. $\mathrm{R}_{f}=0.40$ (75:25 PE/EtOAc; $254 \mathrm{~nm}, \mathrm{I}_{2}$, stains A and C). ${ }^{1} \mathrm{H}-\mathrm{NMR}(500$ $\left.\mathrm{MHz}, \mathrm{CDCl}_{3}\right), \delta: 8.24(2 \mathrm{H}, \mathrm{m}), 7.71(1 \mathrm{H}, \mathrm{m}), 7.55(2 \mathrm{H}, \mathrm{m}), 4.66(1 \mathrm{H}, \mathrm{d}, J=4 \mathrm{~Hz}), 2.40(1 \mathrm{H}$, dsept, $J=4 \mathrm{~Hz}, J=7 \mathrm{~Hz}), 1.19(3 \mathrm{H}, \mathrm{d}, J=7 \mathrm{~Hz}), 0.99(3 \mathrm{H}, \mathrm{d}, J=7 \mathrm{~Hz}) .{ }^{13} \mathrm{C}-\mathrm{NMR}(125 \mathrm{MHz}$, $\left.\mathrm{CDCl}_{3}\right), \delta: 191.4,186.7,135.3,130.1,129.1,125.8,86.1,30.8,18.5,16.0$. IR (neat): 2958, 1746, $1655,1606,1590,1543,1490,1452,1375,1186,1069,1000$, and $712 \mathrm{~cm}^{-1}$. Anal. Calc d for $\mathrm{C}_{12} \mathrm{H}_{13} \mathrm{NO}_{2}$ : C, 70.92; H, 6.45; N, 6.89. Found: C, 71.14; H, 6.26; N,6.87. 


\section{H-5-(2-Methyl)propyl-2-phenyl-2-oxazol-4-one}

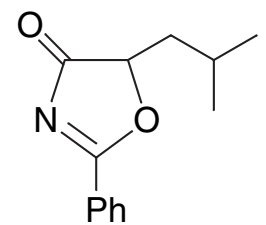

Mp: $56-59$ ¡C. $\mathrm{R}_{f}=0.29$ (85:15 PE/EtOAc; $254 \mathrm{~nm}, \mathrm{I}_{2}$, stains A and C). ${ }^{1} \mathrm{H}-\mathrm{NMR}$ (400 $\left.\mathrm{MHz}, \mathrm{CDCl}_{3}\right), \delta:$ 8.22-8.24 (2H, m), 7.68-7.71 (1H, m), 7.53-7.57 (2H, m), $4.81(1 \mathrm{H}, \mathrm{dd}, J=3.6$, $J=10 \mathrm{~Hz}), 1.91-2.01(2 \mathrm{H}, \mathrm{m}), 1.66-1.73(1 \mathrm{H}, \mathrm{m}), 1.05(6 \mathrm{H}, \mathrm{dd}, J=1.6, J=6.4 \mathrm{~Hz})$.

${ }^{13} \mathrm{C}-\mathrm{NMR}\left(100 \mathrm{MHz}, \mathrm{CDCl}_{3}\right), \delta: 192.5,186.5,135.3,130.2,129.1,126.0,81.0,40.4,25.7,23.0$, 22.1. IR (neat): $3065,2959,2873,1756,1605,1591,1552,1452,1373$, and $1182 \mathrm{~cm}^{-1}$.

\section{H-5-Cyclohexyl-2-phenyl-2-oxazol-4-one}

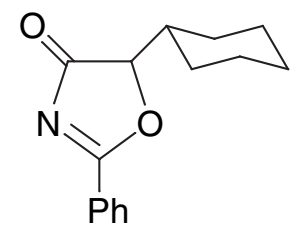

Mp: $106-9$ ¡C. $\mathrm{R}_{f}=0.35$ (85:15 PE/EtOAc; $254 \mathrm{~nm}, \mathrm{I}_{2}$, stains A and C). ${ }^{1} \mathrm{H}-\mathrm{NMR}$ $\left(400 \mathrm{MHz}, \mathrm{CDCl}_{3}\right), \delta: 8.22-8.25(2 \mathrm{H}, \mathrm{m}), 7.69-7.73(1 \mathrm{H}, \mathrm{m}), 7.53-7.58(2 \mathrm{H}, \mathrm{m}), 4.63(1 \mathrm{H}, \mathrm{d}$, $J=4.4 \mathrm{~Hz}), 2.07(1 \mathrm{H}, \mathrm{m}), 1.91-1.94(1 \mathrm{H}, \mathrm{m}), 1.62-1.83(4 \mathrm{H}, \mathrm{m}), 1.14-1.39(5 \mathrm{H}, \mathrm{m}) .{ }^{13} \mathrm{C}-\mathrm{NMR}$ $\left(100 \mathrm{MHz}, \mathrm{CDCl}_{3}\right), \delta: 191.6,168.8,135.3,130.2,129.1,125.9,85.9,40.2,29.0,26.2,25.9,25.7$. IR (neat): 2928, 2854, 1754, 1604, 1590, 1552, 1450, 1364, 1173, and $717 \mathrm{~cm}^{-1}$. Anal. Calc d for $\mathrm{C}_{15} \mathrm{H}_{17} \mathrm{NO}_{2}$ : C, 74.97; H, 7.13; N, 5.83. Found: C, 74.88; H, 7.12; N, 5.76. 


\section{H-5-(2-Propenyl)-2-phenyl oxazol-4-one}

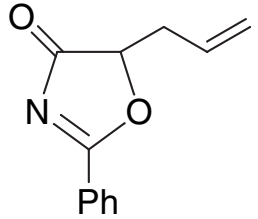

$\mathrm{R}_{f}=0.35\left(8: 2 \mathrm{PE} / \mathrm{EtOAc} ; 254 \mathrm{~nm}, \mathrm{I}_{2}\right.$, stain A). ${ }^{1} \mathrm{H}-\mathrm{NMR}\left(500 \mathrm{MHz}, \mathrm{CDCl}_{3}\right) \delta: 8.22-8.24$ $(2 \mathrm{H}, \mathrm{m}), 7.60-7.63(1 \mathrm{H}, \mathrm{m}), 7.50-7.55(2 \mathrm{H}, \mathrm{m}), 5.77-5.84(1 \mathrm{H}, \mathrm{m}), 5.22(1 \mathrm{H}, \mathrm{ddd}, J=1.2$, $J=2.5, J=17 \mathrm{~Hz}), 5.18(1 \mathrm{H}, \mathrm{dt}, J=1, J=10 \mathrm{~Hz}), 4.86(1 \mathrm{H}, \mathrm{dd}, J=7, J=4.5 \mathrm{~Hz}), 2.84-2.90$ $(1 \mathrm{H}, \mathrm{m}), 2.62-2.68(1 \mathrm{H}, \mathrm{m}) .{ }^{13} \mathrm{C}-\mathrm{NMR}\left(125 \mathrm{MHz}, \mathrm{CDCl}_{3}\right), \delta: 191.3,186.7,135.4,130.3,130.2$, 129.1, 127.9, 125.8, 80.9, 35.2. IR (neat): 3072, 2923, 1757, 1604, 1551, 1451, 1369, 1284, and $1183 \mathrm{~cm}^{-1}$.

\section{General procedure for the preparation of Mo chiral catalyst and setting up of $A A A$ reactions.}

A solution of freshly distilled and degassed ${ }^{2}$ THF $(0.5 \mathrm{~mL})$ of $(R, R)$ [or $(S, S)$ or $( \pm)$, where applied] ligand 5 (4.9 mg, $0.015 \mathrm{mmol})$ and commercialy available $\left[\left(\eta^{3}-\mathrm{C}_{7} \mathrm{H}_{8}\right) \mathrm{Mo}(\mathrm{CO})_{3}(2.8 \mathrm{mg}\right.$, $0.01 \mathrm{mmol}$ ) was gently refluxed under Ar atmosphere at $70 \mathrm{BC}$ for 5 to $10 \mathrm{~min}$ (until deep purple color develops) in a pyrex test tube, sealed with a rubber septum and under Ar atmosphere. To this was added the solution of nucleophile $\mathbf{3}$ (prepared as indicated below), followed by addition of the chosen allylic carbonate $4(0.11 \mathrm{mmol})$. Additions were done via syringe at $60^{\circ} \mathrm{C}$ and the resulting mixture heated at THF reflux (oil bath temperature $70^{\circ} \mathrm{C}$ ) for $8 \mathrm{hr}$. The reaction mixture was then poured onto a $1 \mathrm{~N}$ solution of $1: 1 \mathrm{KH}_{2} \mathrm{PO}_{4} / \mathrm{K}_{2} \mathrm{HPO}_{4}(\mathrm{pH} 7 ; 8 \mathrm{~mL}), \mathrm{DCM}$ was added (15 $\mathrm{mL}$ ). A further extraction with DCM followed, the combined organic layers were finally dried over $\mathrm{MgSO}_{4}$. After elimination of solvents in vacuo, a crude oil was obtained, which after ${ }^{1} \mathrm{H}$ NMR (500 MHz) analysis underwent purification through silica gel (9:1 to 75:25 PE/EtOAc) affording the desired product as a thick oil or white solid, after concentration to dryness on a rotavapor and thourough elimination of solvents in vacuo.

\footnotetext{
${ }^{2}$ Freshly distilled THF was collected in a flame-dried flask and degassed by repeated (three times) freezing in liquid $\mathrm{N}_{2}$, vacuum pumping (pressure in the test tube submitted to degassing was 1 mbar or less) and Ar inflating. The catalyst is very sensitive to oxygen and thus thorough degassing needs to be performed.
} 
Preparation of nucleophile. Test tube containing oxalactim $3(0.1 \mathrm{mmol})$ was evacuated and flushed with argon three times; $0.5 \mathrm{~mL}$ of anhydrous, degassed THF was added, followed by dropwise addition of freshly prepared solution of LHMDS ( $1 \mathrm{M}$ in THF; $0.1 \mathrm{~mL}, 0.1 \mathrm{mmol}$ ) with stirring at $0 i \mathrm{C}$. ${ }^{3}$ The resulting nucleophile was stirred for 5 to $10 \mathrm{~min}$ and directly added to the active catalyst.

\section{H-5-methyl-5-[1-(1-phenyl)prop-3-enyl]-2-phenyl oxazol-4-one.}

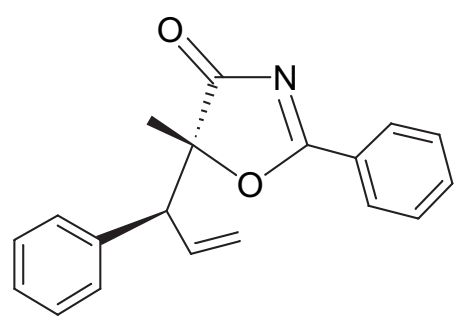

$\mathrm{R}_{f}=0.23\left(9: 1 \mathrm{PE} / \mathrm{EtOAc} ; 254 \mathrm{~nm}, \mathrm{I}_{2}\right.$, stains A, C). ${ }^{1} \mathrm{H}$ NMR $\left(500 \mathrm{MHz}, \mathrm{CDCl}_{3}\right), \delta$ (major diastereomer): 8.23-8.26 (2H, m), 7.7-7.74 $(1 \mathrm{H}, \mathrm{m}), 7.55-7.59(2 \mathrm{H}, \mathrm{m}), 7.28-7.4(5 \mathrm{H}, \mathrm{m}), 5.99$ $(1 \mathrm{H}, \mathrm{ddd}, J=17, J=10, J=8.8 \mathrm{~Hz}), 5.00-5.21(2 \mathrm{H}, \mathrm{m}), 3.79(1 \mathrm{H}, \mathrm{d}, J=8.8 \mathrm{~Hz}), 1.41(3 \mathrm{H}, \mathrm{s})$. ${ }^{13} \mathrm{C}$ NMR (125 MHz, $\mathrm{CDCl}_{3}$ ), $\delta$ (major diastereomer): 193.6, 185.3, 137.8, 135.2, 133.6, 130.0, 129.0, 128.9, 128.7, 127.5, 125.7, 119.8, 90.2, 56.2, 21.2. IR (neat): 2924, 2853, 1754, 1603, $1547,1451,1356,1149$, and $712 \mathrm{~cm}^{-1}$. Anal. Calc d for $\mathrm{C}_{19} \mathrm{H}_{17} \mathrm{NO}_{2}: \mathrm{C}, 78.33 ; \mathrm{H}, 5.88 ; \mathrm{N}, 4.81$; found: $\mathrm{C}, 78.66 ; \mathrm{H}, 6.71 ; \mathrm{N}, 4.02$. Daicel Chiralpak AD column at $\lambda=254 \mathrm{~nm}, 10 \mu \mathrm{L}$ injection at $2 \mathrm{mg} / \mathrm{mL}$, eluent 99:1 Hept/2-PrOH, flow rate $1 \mathrm{~mL} / \mathrm{min}: t_{\mathrm{R}}(1) 27.5 \mathrm{~min}, t_{\mathrm{R}}(2) 34.1 \mathrm{~min}$.

\section{H-5-butyl-5-[1-(1-phenyl)prop-3-enyl]-2-phenyl oxazol-4-one.}

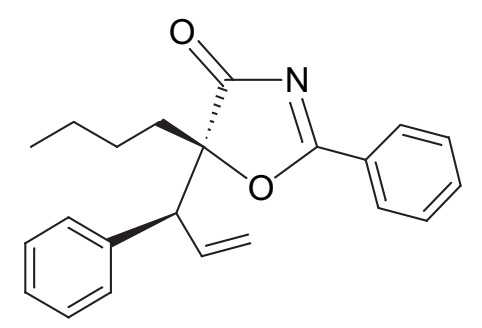

$\mathrm{R}_{f}=0.26\left(9: 1 \mathrm{PE} / \mathrm{EtOAc} ; 254 \mathrm{~nm}, \mathrm{I}_{2}\right.$, stains A, C). ${ }^{1} \mathrm{H}$ NMR (500 MHz, $\left.\mathrm{CDCl}_{3}\right), \delta$ (major diastereomer): 8.23-8.27 (2H, m), 7.68-7.74 (1H, m), 7.45-7.59 (2H, m), 7.24-7.38 (5H, m), 6.01 $(1 \mathrm{H}, \mathrm{ddd}, J=18, J=10, J=8.8 \mathrm{~Hz}), 5.06-5.21(2 \mathrm{H}, \mathrm{m}), 3.8(1 \mathrm{H}, \mathrm{d}, J=8.8 \mathrm{~Hz}), 1.82-1.90$

\footnotetext{
${ }^{3}$ For 5-methyl oxalactim $\left(3, \mathrm{R}=\mathrm{CH}_{3}\right)$ a lower $\mathrm{T}\left(-78^{\circ} \mathrm{C}\right)$ for the preparation of the lithium enolate was preferred.
} 
$(1 \mathrm{H}, \mathrm{m}), 1.62-1.70(1 \mathrm{H}, \mathrm{m}), 1.06-1.21(4 \mathrm{H}, \mathrm{m}), 0.75(3 \mathrm{H}, \mathrm{t}, J=7 \mathrm{~Hz}) .{ }^{13} \mathrm{C}$ NMR $(125 \mathrm{MHz}$, $\mathrm{CDCl}_{3}$ ), $\delta$ (major diastereomer): 193.2, 185.7, 137.9, 135.1, 133.6, 130.0, 129.0, 128.8, 128.7, 127.5, 125.6, 119.6, 93.6, 56.1, 34.5, 24.8, 22.4, 13.7. IR (neat): 2958, 1754, 1604, 1552, 1490, $1452,1358,1152$, and $751 \mathrm{~cm}^{-1}$. Anal. Calc d for $\mathrm{C}_{22} \mathrm{H}_{23} \mathrm{NO}_{2}: \mathrm{C}, 79.25 ; \mathrm{H}, 6.95 ; \mathrm{N}, 4.2$; found: C, 79.12; H, 7.29; N, 4.0. Daicel Chiralpak AD column at $\lambda=254 \mathrm{~nm}, 10 \mu \mathrm{L}$ injection at $2 \mathrm{mg} / \mathrm{mL}$, eluent 95:5 Hept/2-PrOH, flow rate $1 \mathrm{~mL} / \mathrm{min}: t_{\mathrm{R}}$ (1) $9.3 \mathrm{~min}, t_{\mathrm{R}}(2) 10.6 \mathrm{~min}$.

\section{H-5-methyl-5-[1-[1-(2,4-dimethoxyphenyl)]prop-3-enyl]-2-phenyl oxazol-4-one}

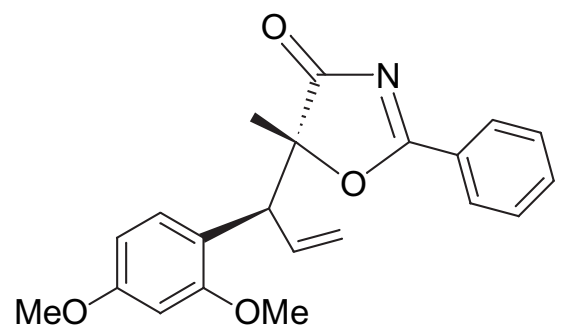

$\mathrm{R}_{f}=0.45$ (8:2 PE/EtOAc; $254 \mathrm{~nm}, \mathrm{I}_{2}$, stains A, C). ${ }^{1} \mathrm{H}-\mathrm{NMR}\left(500 \mathrm{MHz}, \mathrm{CDCl}_{3}\right)$, major diastereoisomer, $\delta:$ 8.23-8.25 $(2 \mathrm{H}, \mathrm{m}), 7.69-7.73(1 \mathrm{H}, \mathrm{m}), 7.53-7.58(2 \mathrm{H}, \mathrm{m}), 7.37(1 \mathrm{H}, \mathrm{d}$, $J=8.5 \mathrm{~Hz}), 6.57(1 \mathrm{H}, \mathrm{dd}, J=8.5, J=2.5 \mathrm{~Hz}), 6.47(1 \mathrm{H}, \mathrm{d}, J=2.5 \mathrm{~Hz}), 5.90$ (1H, ddd, $J=17$, $J=10, J=8.5 \mathrm{~Hz}$ ), 5.17 (1H, ddd, $J=17, J=1.5, J=1 \mathrm{~Hz}), 5.04$ (1H, ddd, $J=10, J=15$, $J=0.5 \mathrm{~Hz}), 4.46(1 \mathrm{H}, \mathrm{d}, J=8.5 \mathrm{~Hz}), 3.83(3 \mathrm{H}, \mathrm{s}), 3.82(3 \mathrm{H}, \mathrm{s}), 1.40(3 \mathrm{H}, \mathrm{s})$.

${ }^{13} \mathrm{C}-\mathrm{NMR}\left(125 \mathrm{MHz}, \mathrm{CDCl}_{3}\right.$ ), major diastereoisomer, $\delta: 194.1,185.2,160.1,158.5,135.2$, 133.9, 130.3, 130.1, 129.7, 129.1, 126.1, 119.5, 118.7, 104.9, 98.6, 91.3, 55.6, 55.5, 20.8. IR (neat): 2936, 2837, 1755, 1605, 1589, 1551, 1506, 1452, 1358, 1209, 1154, 1034, and $710 \mathrm{~cm}^{-1}$. Daicel Chiralpak AD column at $\lambda=254 \mathrm{~nm}, 10 \mu \mathrm{L}$ injection at $2 \mathrm{mg} / \mathrm{mL}$, eluent 98:2 Hept/2-PrOH, flow rate $1 \mathrm{~mL} / \mathrm{min}: t_{\mathrm{R}}$ (1) $27.3 \mathrm{~min}, t_{\mathrm{R}}(2) 29.6 \mathrm{~min}$. 


\section{H-5-methyl-5-[1-[1-(2-bromophenyl)]prop-3-enyl]-2-phenyl oxazol-4-one}

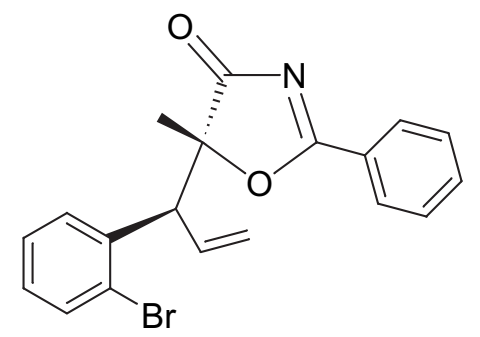

$\mathrm{R}_{f}=0.28\left(9: 1 \mathrm{PE} / \mathrm{EtOAc} ; 254 \mathrm{~nm}, \mathrm{I}_{2}\right.$, stains A, C). ${ }^{1} \mathrm{H}$ NMR $\left(500 \mathrm{MHz}, \mathrm{CDCl}_{3}\right), \delta$ (major diastereomer): 8.23-8.27 (2H, m), 7.70-7.74 (1H, m), 7.54-7.61 $(4 \mathrm{H}, \mathrm{m}), 7.38(1 \mathrm{H}, \mathrm{dt}, J=7.8$, $J=1.2 \mathrm{~Hz}), 7.11-7.16(1 \mathrm{H}, \mathrm{m}), 5.91(1 \mathrm{H}, \mathrm{ddd}, J=17, J=10, J=8.5 \mathrm{~Hz}), 5.10-5.27(2 \mathrm{H}, \mathrm{m})$, $4.61(1 \mathrm{H}, \mathrm{d}, J=8.5 \mathrm{~Hz}), 1.47(3 \mathrm{H}, \mathrm{s}) .{ }^{13} \mathrm{C} \mathrm{NMR}\left(125 \mathrm{MHz}, \mathrm{CDCl}_{3}\right), \delta$ (major diastereomer): 193.1, 185.2, 137.2, 135.2, 133.3, 132.5, 130.0, 129.4, 129.0, 128.9, 127.8, 125.8, 125.6, 120.6, 90.3, 53.8, 20.9. IR (neat): 2926, 1756, 1604, 1548, 1451, 1356, 1153, and $707 \mathrm{~cm}^{-1}$. Anal. Calc d for $\mathrm{C}_{19} \mathrm{H}_{16} \mathrm{BrNO}_{2}$ : C, 61.64; H, 4.36; N, 3.78; found: C, 61.86; H, 4.37; N, 3.68. Daicel Chiralpak AD column at $\lambda=254 \mathrm{~nm}, 10 \mu \mathrm{L}$ injection at $2 \mathrm{mg} / \mathrm{mL}$, eluent 99:1 Hept/2PrOH, flow rate $1 \mathrm{~mL} / \mathrm{min}: t_{\mathrm{R}}$ (1) $26.2 \mathrm{~min}, t_{\mathrm{R}}$ (2) $30.3 \mathrm{~min}$.

\section{H-5-methyl-5-[1-(1-cyclohexenyl)prop-3-enyl]-2-phenyl oxazol-4-one}

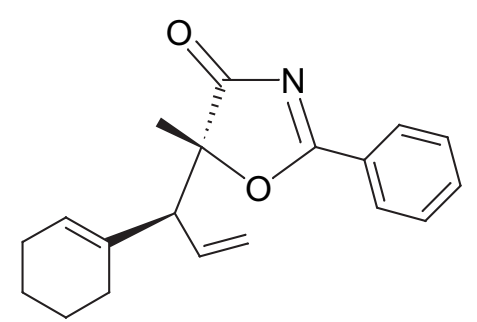

$\mathrm{R}_{f}=0.72\left(8: 2 \mathrm{PE} / \mathrm{EtOAc} ; 254 \mathrm{~nm}, \mathrm{I}_{2}\right.$, stains A, C). ${ }^{1} \mathrm{H}-\mathrm{NMR}\left(500 \mathrm{MHz}, \mathrm{CDCl}_{3}\right)$, major diastereoisomer, $\delta$ : 8.21-8.23 (2H, m), 7.68-7.73 (1H, m), 7.54-7.58 (2H, m), $5.75(1 \mathrm{H}$, ddd, $J=16, J=10, J=8.8 \mathrm{~Hz}), 5.71(1 \mathrm{H}, \mathrm{m}), 5.16(1 \mathrm{H}, \mathrm{dt}, J=16, J=0.8 \mathrm{~Hz}), 5.04(1 \mathrm{H}, \mathrm{dd}, J=10$, $J=0.8 \mathrm{~Hz}), 3.13(1 \mathrm{H}, \mathrm{d}, J=8.8 \mathrm{~Hz}), 2.03-2.10(4 \mathrm{H}, \mathrm{m}), 1.55-1.62(2+2 \mathrm{H}, \mathrm{m}+\mathrm{m}), 1.51(3 \mathrm{H}, \mathrm{s})$. ${ }^{13} \mathrm{C}-\mathrm{NMR}\left(125 \mathrm{MHz}, \mathrm{CDCl}_{3}\right.$ ), major diastereoisomer, $\delta:$ 194.3, 185.3, 135.2, 134.5, 132.7, 130.1, 129.1, 127.7, 126.1, 119.3, 90.4, 58.3, 26.9, 25.6, 23.1, 22.4, 21.1. IR (neat): 2929, 1757, $1065,1591,1552,1451,1356$, and $1140 \mathrm{~cm}^{-1}$. Anal. Calc d for $\mathrm{C}_{19} \mathrm{H}_{21} \mathrm{NO}_{2}: \mathrm{C}, 77.26 ; \mathrm{H}, 7.17$, $\mathrm{N}, 4.74$. Found: C, 77.12; H, 6.89, N, 4.75. Daicel Chiralpak AD column at $\lambda=254 \mathrm{~nm}, 10 \mu \mathrm{L}$ 
injection at $2 \mathrm{mg} / \mathrm{mL}$, eluent 99:1 Hept/2-PrOH, flow rate $1 \mathrm{~mL} / \mathrm{min}: t_{\mathrm{R}}$ (1) $17.7 \mathrm{~min}$, $t_{\mathrm{R}}(2) 21.9 \mathrm{~min}$.

\section{H-5-methyl-5-[3-(5-methyl)hexa-1,4-dienyl]-2-phenyl oxazol-4-one}

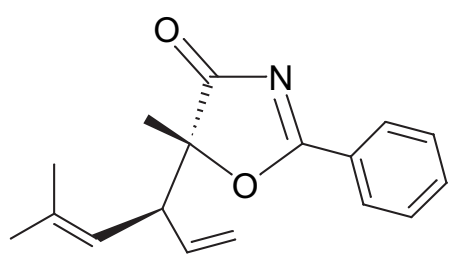

$\mathrm{R}_{f}=0.67\left(8: 2 \mathrm{PE} / \mathrm{EtOAc} ; 254 \mathrm{~nm}, \mathrm{I}_{2}\right.$, stains A, C). ${ }^{1} \mathrm{H}-\mathrm{NMR}\left(400 \mathrm{MHz}, \mathrm{CDCl}_{3}\right)$, major diastereoisomer, $\delta$ : 8.21-8.23 $(2 \mathrm{H}, \mathrm{m}), 7.67-7.72(1 \mathrm{H}, \mathrm{m}), 7.52-7.56(2 \mathrm{H}, \mathrm{m}), 5.64(1 \mathrm{H}$, ddd, $J=16, J=10, J=8 \mathrm{~Hz}), 5.20(1 \mathrm{H}, \mathrm{dt}, J=10, J=1.4 \mathrm{~Hz}), 5.15(1 \mathrm{H}, \mathrm{dd}, J=16, J=1.2 \mathrm{~Hz}), 5.03$ $(1 \mathrm{H}, \mathrm{m}), 3.47(1 \mathrm{H}, \mathrm{d}, J=8, J=0.8 \mathrm{~Hz}), 1.76(3 \mathrm{H}, \mathrm{d}, J=1.2 \mathrm{~Hz}), 1.67(3 \mathrm{H}, \mathrm{d}, J=1.2 \mathrm{~Hz}), 1.56$ $(3 \mathrm{H}, \mathrm{s}) .{ }^{13} \mathrm{C}-\mathrm{NMR}\left(100 \mathrm{MHz}, \mathrm{CDCl}_{3}\right)$, major diastereoisomer, $\delta: 193.89,185.4,137.1,135.1$, 133.7, 130.1, 129.0, 126.1, 119.7, 118.5, 90.3, 49.2, 26.1, 20.8, 18.3. IR (neat): 3072, 2979, 2931, 1758, 1605, 1590, 1552, 1490, 1451, 1357, 1153, 1036, and $713 \mathrm{~cm}^{-1}$. Anal. Calc d for $\mathrm{C}_{17} \mathrm{H}_{19} \mathrm{NO}_{2}$ : C, 75.81; H, 7.11, N, 5.20. Found: C, 75.60; H, 6.83, N, 5.10. Daicel Chiralpak AD column at $\lambda=254 \mathrm{~nm}, 10 \mu \mathrm{L}$ injection at $2 \mathrm{mg} / \mathrm{mL}$, eluent 99.5:0.5 Hept/2-PrOH, flow rate $1 \mathrm{~mL} / \mathrm{min}: t_{\mathrm{R}}(1) 24.5 \mathrm{~min}, t_{\mathrm{R}}$ (2) $28.9 \mathrm{~min}$.

\section{H-5-(3-propenyl)-5-[1-(1-phenyl)prop-3-enyl]-2-phenyl oxazol-4-one}

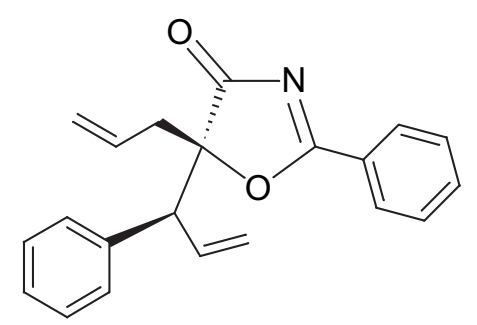

$\mathrm{R}_{f}=0.65\left(8: 2 \mathrm{PE} / \mathrm{EtOAc} ; 254 \mathrm{~nm}, \mathrm{I}_{2}\right.$, stains A, C). ${ }^{1} \mathrm{H}-\mathrm{NMR}\left(500 \mathrm{MHz}, \mathrm{CDCl}_{3}\right)$, major diastereoisomer, $\delta$ : $8.22(2 \mathrm{H}, \mathrm{dd}, J=8 \mathrm{~Hz}, J=1 \mathrm{~Hz}), 7.69-7.73(1 \mathrm{H}, \mathrm{m}), 7.56(2 \mathrm{H}, \mathrm{t}, J=8 \mathrm{~Hz})$, 7.36-7.38 (4H, m), 7.27-7.30 (1H, m), $6.02(1 \mathrm{H}, \mathrm{ddd}, J=10, J=8, J=7 \mathrm{~Hz}), 5.49-5.57(1 \mathrm{H}, \mathrm{m})$, $5.19(1 \mathrm{H}, \mathrm{d}, J=17 \mathrm{~Hz}), 5.11(1 \mathrm{H}, \mathrm{d}, J=10 \mathrm{~Hz}), 5.06(1 \mathrm{H}, \mathrm{dd}, J=17, J=1.5 \mathrm{~Hz}), 5.01(1 \mathrm{H}, \mathrm{dd}$, $J=10.5, J=1 \mathrm{~Hz}), 3.84(1 \mathrm{H}, \mathrm{d}, J=9 \mathrm{~Hz}), 2.59(1 \mathrm{H}, \mathrm{dd}, J=7, J=14 \mathrm{~Hz}), 2.41(1 \mathrm{H}, \mathrm{dd}, J=7.5$, $J=14 \mathrm{~Hz}) .{ }^{13} \mathrm{C}-\mathrm{NMR}\left(125 \mathrm{MHz}, \mathrm{CDCl}_{3}\right)$, major diastereoisomer, $\delta: 192.7,185.7,137.8,135.3$, 
133.6, 130.1, 129.2, 129.1, 129.1, 128.9, 127.8, 1254.7, 121.3, 120.0, 92.7, 55.8, 39.2.

IR (neat): 3064, 1755, 1604, 1551, 1452, 1359, 725, and $699 \mathrm{~cm}^{-1}$. Daicel Chiralpak AD column at $\lambda=254 \mathrm{~nm}, 10 \mu \mathrm{L}$ injection at $2 \mathrm{mg} / \mathrm{mL}$, eluent 99:1 Hept $/ 2-\mathrm{PrOH}$, flow rate $1 \mathrm{~mL} / \mathrm{min}$ : $t_{\mathrm{R}}(1) 26.9 \mathrm{~min}, t_{\mathrm{R}}(2) 30.9 \mathrm{~min}$.

5H-3 -Phenyl-spiro[(2-phenyloxazol-4-one)-5,4 -cyclopentene] or 9-oxo-8-phenyl-3oxa-1-aza-spiro[4.4]nona-1,6-diene

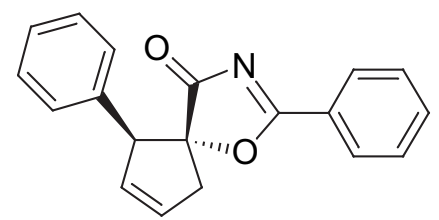

$\mathrm{R}_{f}=0.3\left(85: 15\right.$ PE/EtOAc; $\mathrm{I}_{2}$, stains A, C). ${ }^{1} \mathrm{H}-\mathrm{NMR}\left(500 \mathrm{MHz}, \mathrm{CDCl}_{3}\right), \delta: 8.24(2 \mathrm{H}, \mathrm{dt}$, $J=8, J=0.5 \mathrm{~Hz}), 7.70(1 \mathrm{H}, \mathrm{t}, J=7.5 \mathrm{~Hz}), 7.55(2 \mathrm{H}, \mathrm{dd}, J=7.5, J=8 \mathrm{~Hz}), 7.25-7.30(3 \mathrm{H}, \mathrm{m})$, 7.09-7.11 (2H, m), $6.13(1 \mathrm{H}, \mathrm{dt}, J=4, J=2 \mathrm{~Hz}), 5.94(1 \mathrm{H}, \mathrm{dt}, J=4, J=2 \mathrm{~Hz}), 4.42(1 \mathrm{H}, \mathrm{s}), 3.12$ $(1 \mathrm{H}, \mathrm{dt}, J=15, J=2 \mathrm{~Hz}), 2.91(1 \mathrm{H}, \mathrm{dt}, J=17, J=2 \mathrm{~Hz}) \cdot{ }^{13} \mathrm{C}-\mathrm{NMR}\left(125 \mathrm{MHz}, \mathrm{CDCl}_{3}\right)$, $\delta: 190.5,184.9,135.8,135.2,131.3,130.2,129.5,129.1,128.6,128.5,128.0,126.1,94.8,61.5$, 42.6. IR (neat): 3062, 2924, 1757, 1604, 1590, 1552, 1451, 1355, 1035, and $697 \mathrm{~cm}^{-1}$.

\section{H-5-(2-methyl)propyl-5-[1-(2-thienyl)prop-3-enyl]-2-phenyl oxazol-4-one}

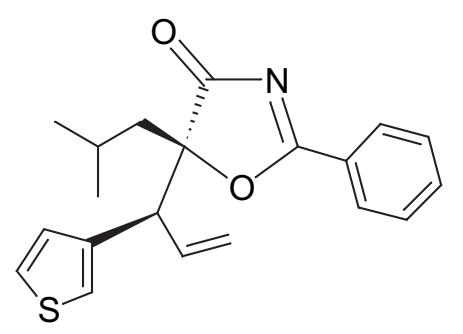

$\mathrm{R}_{f}=0.75\left(8: 2 \mathrm{PE} / \mathrm{EtOAc} ; 254 \mathrm{~nm}, \mathrm{I}_{2}\right.$, stains A, C). ${ }^{1} \mathrm{H}-\mathrm{NMR}$ (400 MHz, $\mathrm{CDCl}_{3}$ ), major diastereoisomer, $\delta:$ 8.19-8.22 $(2 \mathrm{H}, \mathrm{m}), 7.69-7.73(1 \mathrm{H}, \mathrm{m}), 7.54-7.58(2 \mathrm{H}, \mathrm{m}), 7.15(1 \mathrm{H}, \mathrm{dd}$, $J=1.4 \mathrm{~Hz}, J=3 \mathrm{~Hz}), 7.06(1 \mathrm{H}, \mathrm{dd}, J=1.4, J=3 \mathrm{~Hz}), 6.02(1 \mathrm{H}, \mathrm{ddd}, J=8.8, J=10 \mathrm{~Hz}$, $J=17 \mathrm{~Hz}), 5.16(1 \mathrm{H}, \mathrm{dt}, J=1.2, J=17 \mathrm{~Hz}), 5.11(1 \mathrm{H}, \mathrm{dt}, J=10, J=0.6 \mathrm{~Hz}), 3.95(1 \mathrm{H}, \mathrm{d}$, $J=8.8 \mathrm{~Hz}), 1.85-1.90(1 \mathrm{H}, \mathrm{m}), 1.72-1.78(1 \mathrm{H}, \mathrm{m}), 1.58-1.63(1 \mathrm{H}, \mathrm{m}), 0.85(3 \mathrm{H}, \mathrm{d}, J=6.4) .0 .78$ $(3 \mathrm{H}, \mathrm{d}, J=6.8) .{ }^{13} \mathrm{C}-\mathrm{NMR}\left(100 \mathrm{MHz}, \mathrm{CDCl}_{3}\right)$, major diastereoisomer, $\delta: 193.3,185.8,138.0$, 135.3, 133.3, 130.1, 129.2, 127.7, 126.1, 125.9, 123.2, 119.7, 93.2, 52.2, 43.3, 24.3, 24.2, 24.0. IR (neat): 3088, 2958, 2872, 1753, 1604, 1590, 1551, 1490, 1451, 1357, and $715 \mathrm{~cm}^{-1}$. 
Anal. Calc d for $\mathrm{C}_{20} \mathrm{H}_{21} \mathrm{NO}_{2} \mathrm{~S}: \mathrm{C}, 70.77 ; \mathrm{H}, 6.24$. Found: C, 71.14; H, 6.16. Daicel Chiralpak AD column at $\lambda=254 \mathrm{~nm}, 10 \mu \mathrm{L}$ injection at $2 \mathrm{mg} / \mathrm{mL}$, eluent 99:1 Hept/2-PrOH, flow rate $1 \mathrm{~mL} / \mathrm{min}: t_{\mathrm{R}}(1) 12.7 \mathrm{~min}, t_{\mathrm{R}}(2) 30.7 \mathrm{~min}$.

\section{H-5-(methyl)ethyl-5-[1-(1-phenyl)prop-3-enyl]-2-phenyl oxazol-4-one}

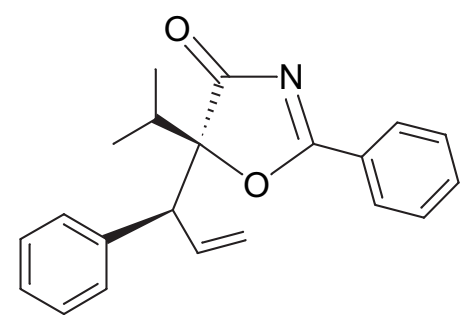

$\mathrm{R}_{f}=0.69$ (8:2 PE/EtOAc; $254 \mathrm{~nm}, \mathrm{I}_{2}$, stains A, C). ${ }^{1} \mathrm{H}-\mathrm{NMR}\left(400 \mathrm{MHz}, \mathrm{CDCl}_{3}\right)$, major diastereoisomer, $\delta$ : 8.18-8.21 $(2 \mathrm{H}, \mathrm{m})$, 7.68-7.71 $(1 \mathrm{H}, \mathrm{m}), 7.53-7.56(2 \mathrm{H}, \mathrm{m}), 7.36-7.38(2 \mathrm{H}, \mathrm{m})$, 7.22-7.31 (3H, m), 6.12 (1H, ddd, $J=17, J=10, J=8.8 \mathrm{~Hz}), 5.17(1 \mathrm{H}, \mathrm{d}, J=17 \mathrm{~Hz}), 5.12$ $(1 \mathrm{H}, \mathrm{dt}, J=10, J=1 \mathrm{~Hz}), 4.01(1 \mathrm{H}, \mathrm{d}, J=8.8 \mathrm{~Hz}), 2.20(1 \mathrm{H}$, sept, $J=6.8 \mathrm{~Hz}), 1.02(3 \mathrm{H}, \mathrm{d}$, $J=6.8 \mathrm{~Hz}), 0.94(3 \mathrm{H}, \mathrm{d}, J=6.8 \mathrm{~Hz}) .{ }^{13} \mathrm{C}-\mathrm{NMR}\left(100 \mathrm{MHz}, \mathrm{CDCl}_{3}\right)$, major diastereoisomer, $\delta$ : 192.6, 185.7, 138.1, 135.2, 130.0, 129.1, 128.8, 127.6, 125.7, 119.5, 95.6, 53.7, 32.1, 16.2, 15.6. IR (neat): 2973, 1750, 1604, 1591, 1552, 1490, 1451, and $1356 \mathrm{~cm}^{-1}$. Daicel Chiralpak AD column at $\lambda=254 \mathrm{~nm}, 10 \mu \mathrm{L}$ injection at $2 \mathrm{mg} / \mathrm{mL}$, eluent 99:1 Hept $/ 2-\mathrm{PrOH}$, flow rate $1 \mathrm{~mL} / \mathrm{min}: t_{\mathrm{R}}(1) 21.6 \mathrm{~min}, t_{\mathrm{R}}(2) 28.6 \mathrm{~min}$.

\section{H-5-cyclohexyl-5-[1-(1-phenyl)prop-3-enyl]-2-phenyl oxazol-4-one}

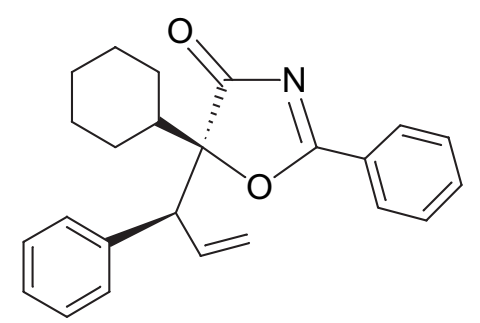

$\mathrm{R}_{f}=0.67$ (8:2 PE/EtOAc; $254 \mathrm{~nm}, \mathrm{I}_{2}$, stains A, C). ${ }^{1} \mathrm{H}-\mathrm{NMR}\left(500 \mathrm{MHz}, \mathrm{CDCl}_{3}\right)$, major diastereoisomer, $\delta:$ 8.18-8.20 $(2 \mathrm{H}, \mathrm{m}), 7.67-7.71(1 \mathrm{H}, \mathrm{m}), 7.52-7.56(2 \mathrm{H}, \mathrm{m}), 7.34-7.36(2 \mathrm{H}, \mathrm{m})$, 7.26-7.30 (2H, m), 7.20-7.23 (1H, m), $6.14(1 \mathrm{H}, \mathrm{ddd}, J=17, J=10, J=9 \mathrm{~Hz}), 5.18(1 \mathrm{H}, \mathrm{dt}$, $J=16, J=1 \mathrm{~Hz}), 5.12(1 \mathrm{H}, \mathrm{dd}, J=10, J=1 \mathrm{~Hz}), 4.03(1 \mathrm{H}, \mathrm{d}, J=9 \mathrm{~Hz}), 1.84-1.89(1 \mathrm{H}, \mathrm{m})$, 1.75-1.77 (1H, m), 1.66-1.70 (3H, m), 1.57 (1H, m), 1.36-1.41 (1H, m), 0.99-1.10 (4H, m). 
${ }^{13} \mathrm{C}-\mathrm{NMR}\left(125 \mathrm{MHz}, \mathrm{CDCl}_{3}\right.$ ), major diastereoisomer, $\delta:$ 192.8, 185.6, 138.0, 135.1, 134.1, 130.0, 129.9, 129.1, 128.7, 127.5, 125.7, 119.5, 95.4, 53.0, 41.3, 26.1, 26.0, 25.9, 25.9, 25.3. IR (neat): 3063, 6061, 2933, 2855, 1750, 1604, 1591, 1552, 1490, 1451, 1359, and $721 \mathrm{~cm}^{-1}$. Anal. Calc d for $\mathrm{C}_{24} \mathrm{H}_{25} \mathrm{NO}_{2}$ : C, 80.19; H, 7.01, N, 3.90. Found: C, 79.86; H, 6.98, N, 3.90. Daicel Chiralpak AD column at $\lambda=254 \mathrm{~nm}, 10 \mu \mathrm{L}$ injection at $2 \mathrm{mg} / \mathrm{mL}$, eluent 99:1 Hept/2-PrOH, flow rate $1 \mathrm{~mL} / \mathrm{min}: t_{\mathrm{R}}$ (1) $30.2 \mathrm{~min}, t_{\mathrm{R}}(2) 52.2 \mathrm{~min}$.

\section{H-5-methylphenyl-5-[1-(1-phenyl)prop-3-enyl]-2-phenyl oxazol-4-one}

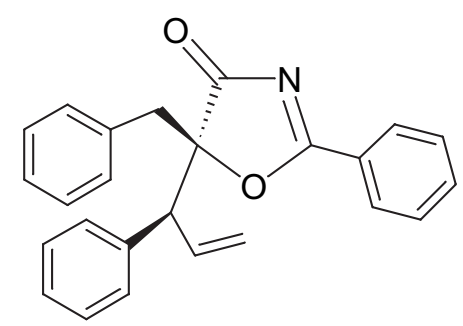

Mp: $111-118$ ¡C (softening starts at 97 ¡C). $\mathrm{R}_{f}=0.66$ (8:2 PE/EtOAc; $254 \mathrm{~nm}, \mathrm{I}_{2}$, stains A, C). ${ }^{1} \mathrm{H}-\mathrm{NMR}\left(400 \mathrm{MHz}, \mathrm{CDCl}_{3}\right.$ ), major diastereoisomer, $\delta$ : 8.04-8.06 (2H, m), 7.62-7.66 $(1 \mathrm{H}, \mathrm{m}), 7.36-7.50(6 \mathrm{H}, \mathrm{m}), 7.28-7.32(1 \mathrm{H}, \mathrm{m}), 7.06(5 \mathrm{H}, \mathrm{m}), 6.05(1 \mathrm{H}, \mathrm{ddd}, J=17, J=10$, $J=8.8 \mathrm{~Hz}), 5.20(1 \mathrm{H}, \mathrm{dt}, J=17, J=0.8 \mathrm{~Hz}), 5.13(1 \mathrm{H}, \mathrm{dd}, J=10, J=0.8 \mathrm{~Hz}), 3.94(1 \mathrm{H}, \mathrm{d}$, $J=8.8 \mathrm{~Hz}), 3.14(1 \mathrm{H}, \mathrm{d}, J=14 \mathrm{~Hz}), 2.96(1 \mathrm{H}, \mathrm{d}, J=14 \mathrm{~Hz}) .{ }^{13} \mathrm{C}-\mathrm{NMR}\left(100 \mathrm{MHz}, \mathrm{CDCl}_{3}\right)$, major diastereoisomer, $\delta$ : 192.5, 185.5, 138.1, 135.1, 133.7, 133.2, 130.2, 130.1, 129.9, 129.7, 129.1, 129.0, 128.9, 128.8, 128.7, 128.4, 128.3, 127.8, 127.4, 125.6, 120.1, 93.3, 56.2, 41.1. IR (neat): $3031,1753,1604,1589,1550,1452$, and $1360 \mathrm{~cm}^{-1}$. Anal. Calc d for $\mathrm{C}_{25} \mathrm{H}_{21} \mathrm{NO}_{2}$ : C, 81.72; H, 5.76, N, 3.81. Found: C, 82.12; H, 5.91, N, 3.81. Daicel Chiralpak AD column at $\lambda=254 \mathrm{~nm}, 10 \mu \mathrm{L}$ injection at $2 \mathrm{mg} / \mathrm{mL}$, eluent 98:2 Hept $/ 2-\mathrm{PrOH}$, flow rate $1 \mathrm{~mL} / \mathrm{min}$ : $t_{\mathrm{R}}(1) 39.6 \mathrm{~min}, t_{\mathrm{R}}(2) 45.3 \mathrm{~min}$. 


\section{General procedure for the hydrolysis to tertiary $\alpha$-hydroxy carboxyamides.}

To the 5,5-dialkylated oxazol-4-one $6(0.1 \mathrm{mmol}) 2.5 \mathrm{~N} \mathrm{NaOH}(0.05 \mathrm{~mL})$, and $\mathrm{EtOH}$ $(0.2 \mathrm{~mL})$ were added. The mixture was stirred for $30 \mathrm{~min}$ to $3 \mathrm{hr}$ at $60 i \mathrm{C}$, after which the reaction was deemed complete from TLC. After correction of $\mathrm{pH}$ into acidic with $1 \mathrm{~N} \mathrm{NaHSO}_{4}$, extraction with $\mathrm{CHCl}_{3}$ followed. The combined organic layers were then dried over $\mathrm{MgSO}_{4}$. After elimination of solvents in vacuo, a crude oil was obtained, which underwent purification through silica gel (1:1 PE/EtOAc, 2\% HOAc) affording the expectd product, commonly as a white solid, after concentration to dryness on a rotavapor and thourough elimination of solvents in vacuo.

\section{2-Hydroxy-2-methyl-3-phenylpent-4-enamide}

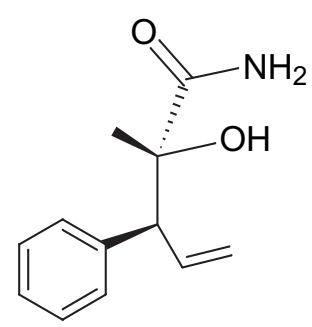

${ }^{1} \mathrm{H}-\mathrm{NMR}\left(500 \mathrm{MHz}, \mathrm{CDCl}_{3}\right), \delta$ (major diastereomer): 7.25-7.38 $(5 \mathrm{H}, \mathrm{m}), 6.70(1 \mathrm{H}, \mathrm{br} \mathrm{s})$, $6.28(1 \mathrm{H}, \mathrm{ddd}, J=17, J=10, J=8.5 \mathrm{~Hz}), 5.62(1 \mathrm{H}, \mathrm{br} \mathrm{s}), 5.12-5.19(2 \mathrm{H}, \mathrm{m}), 3.78(1 \mathrm{H}, \mathrm{d}$, $J=8.5 \mathrm{~Hz}), 2.29(1 \mathrm{H}, \mathrm{br} \mathrm{s}), 1.26(3 \mathrm{H}, \mathrm{s}) .{ }^{13} \mathrm{C}-\mathrm{NMR}\left(125 \mathrm{MHz}, \mathrm{CDCl}_{3}\right), \delta$ (major diastereomer): 177.7, 139.0, 136.2, 129.4, 128.6, 127.2, 118.2, 78.2, 57.0, 25.7. IR (neat): 3466, 3344, 2926, $1665,1572,1453,1369,1120,925$, and $702 \mathrm{~cm}^{-1}$.

\section{2-Hydroxy-2-methyl-3-(2-bromophenyl)pent-4-enamide}

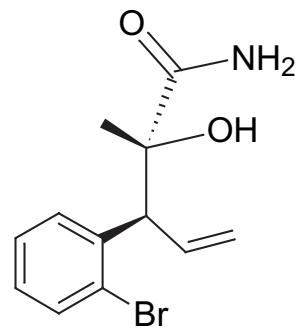

${ }^{1} \mathrm{H}-\mathrm{NMR}\left(500 \mathrm{MHz}, \mathrm{CDCl}_{3}\right), \delta$ (major diastereomer): 7.56-7.61 $(2 \mathrm{H}, \mathrm{m}), 7.30(1 \mathrm{H}, \mathrm{dt}$, $J=7.6, J=1.5 \mathrm{~Hz}), 7.05-7.14(1 \mathrm{H}, \mathrm{m}), 6.72(1 \mathrm{H}, \mathrm{br} \mathrm{s}), 6.59(1 \mathrm{H}, \mathrm{br} \mathrm{s}), 6.16(1 \mathrm{H}, \mathrm{ddd}, J=17$, 
$J=10, J=8.5 \mathrm{~Hz}), 5.11-5.17(2 \mathrm{H}, \mathrm{m}), 4.480(1 \mathrm{H}, \mathrm{d}, J=8.5 \mathrm{~Hz}), 1.30(3 \mathrm{H}, \mathrm{s})$.

${ }^{13} \mathrm{C}-\mathrm{NMR}\left(125 \mathrm{MHz}, \mathrm{CDCl}_{3}\right.$ ), $\delta$ (major diastereomer): 179.0, 139.0, 135.7, 133.0, 130.3, 128.4, 127.4, 126.2, 118.6, 78.3, 54.4, 24.9.IR (neat): 3467, 3346, 2977, 1671, 1565, 1469, 1370, 1114, 926 , and $751 \mathrm{~cm}^{-1}$.

\section{2-Hydroxy-2-(2-methyhl)propyl-3-(2-thienyl)pent-4-enamide}

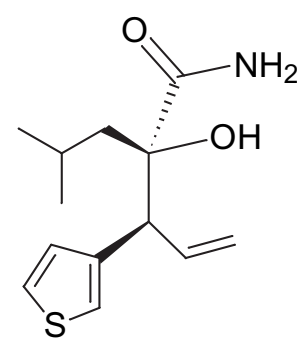

Mp: 123-124 ¡C (softening starts at $115\lceil\mathrm{C}) . \mathrm{R}_{f}=0.6\left(1: 1 \mathrm{PE} / \mathrm{EtOAc}, 2 \% \mathrm{HOAc} \mathrm{I}_{2}\right.$, stains A, C). ${ }^{1} \mathrm{H}-\mathrm{NMR}\left(500 \mathrm{MHz}, \mathrm{CDCl}_{3}\right), \delta: 7.32(1 \mathrm{H}, \mathrm{dd}, J=5 \mathrm{~Hz}, J=3 \mathrm{~Hz}), 7.12(1 \mathrm{H}, \mathrm{dd}, J=3$, $J=1.5 \mathrm{~Hz}), 7.06(1 \mathrm{H}, \mathrm{dt}, J=3, J=1.5 \mathrm{~Hz}), 6.66(1 \mathrm{H}, \mathrm{br} \mathrm{s}), 6.18(1 \mathrm{H}, \mathrm{ddd}, J=17, J=10$, $J=8.5 \mathrm{~Hz}), 5.93(1 \mathrm{H}, \mathrm{br} \mathrm{s}), 5.13(1 \mathrm{H}, \mathrm{ddd}, J=10 \mathrm{~Hz}, J=1.5 \mathrm{~Hz}, J=0.5 \mathrm{~Hz}), 5.09(1 \mathrm{H}$, ddd, $J=17, J=1.5, J=1 \mathrm{~Hz}), 3.84(1 \mathrm{H}, \mathrm{d}, J=8.5 \mathrm{~Hz}), 2.31(1 \mathrm{H}, \mathrm{br} \mathrm{s}), 1.84(1 \mathrm{H}, \mathrm{dd}, J=5$, $J=9 \mathrm{~Hz}), 1.74(1 \mathrm{H}$, sept, $J=7 \mathrm{~Hz}), 1.12(1 \mathrm{H}, \mathrm{dd}, J=7, J=14.5 \mathrm{~Hz}), 0.89(3 \mathrm{H}, \mathrm{d}, J=7 \mathrm{~Hz})$, $0.86(3 \mathrm{H}, \mathrm{d}, J=7 \mathrm{~Hz}) .{ }^{13} \mathrm{C}-\mathrm{NMR}\left(125 \mathrm{MHz}, \mathrm{CDCl}_{3}\right), \delta: 177.0,139.3,136.1,128.5,125.9,122.7$, 118.0, 81.0, 54.3, 46.6, 24.6, 24.2, 24.1. IR (neat): 3480, 3282, 3084, 2955, 2872, 1673, 1578, $1470,1416,1322,1270,1144$, and $1036 \mathrm{~cm}^{-1}$.

\section{2-Hydroxy-2-(methyl)ethyl-3-phenylpent-4-enamide}

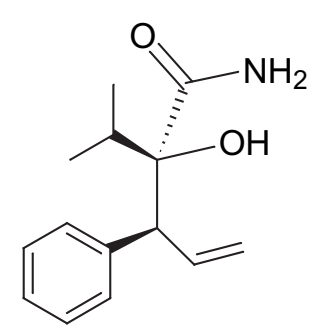

Mp: 98-101 ¡C. $\mathrm{R}_{f}=0.65$ (1:1 PE/EtOAc, 2\% HOAc; $\mathrm{I}_{2}$, stains A, C). ${ }^{1} \mathrm{H}-\mathrm{NMR}(500 \mathrm{MHz}$, $\left.\mathrm{CDCl}_{3}\right), \delta: 7.26-7.35(5 \mathrm{H}, \mathrm{m}) 6.46(1 \mathrm{H}, \mathrm{ddd}, J=17 \mathrm{~Hz}, J=9.5 \mathrm{~Hz}, J=8.5 \mathrm{~Hz}), 6.42(1 \mathrm{H}, \mathrm{br}$ s), $5.56(1 \mathrm{H}, \mathrm{br} \mathrm{s}), 5.16(1 \mathrm{H}, \mathrm{dt}, J=9.5, J=1 \mathrm{~Hz}), 5.09(1 \mathrm{H}, \mathrm{dt}, J=17, J=1 \mathrm{~Hz}), 3.80(1 \mathrm{H}, \mathrm{d}$, $J=8.5 \mathrm{~Hz}), 2.26(1 \mathrm{H}, \mathrm{br} \mathrm{s}), 2.01(1 \mathrm{H}, \mathrm{sept}, J=7 \mathrm{~Hz}), 0.99(3 \mathrm{H}, \mathrm{d}, J=7 \mathrm{~Hz}), 0.97(3 \mathrm{H}, \mathrm{d}$, 
$J=7 \mathrm{~Hz}) .{ }^{13} \mathrm{C}-\mathrm{NMR}\left(125 \mathrm{MHz}, \mathrm{CDCl}_{3}\right), \delta: 176.3,139.4,137.3,129.5,128.7,127.3,117.9,82.7$, 55.7, 33.4, 17.8, 15.9. IR (neat): 3483, 3384, 2970, 1668, 1564, 1156, and $7021 \mathrm{~cm}^{-1}$.

\section{1-Hydroxy-2-phenylcyclopent-3-enylcarboxamide}

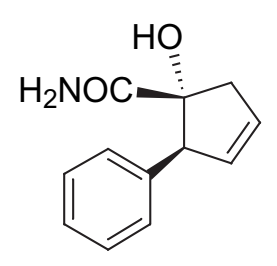

$\mathrm{R}_{f}=0.3\left(1: 1 \mathrm{PE} / \mathrm{EtOAc}, 2 \%\right.$ HOAc; $\mathrm{I}_{2}$, stains A, C). ${ }^{1} \mathrm{H}-\mathrm{NMR}\left(500 \mathrm{MHz}, \mathrm{CDCl}_{3}\right), \delta$ :

7.16-7.30 (5H, m), 6.07 (1H, ddd, $J=2 \mathrm{~Hz}, J=4 \mathrm{~Hz}, J=6 \mathrm{~Hz}), 5.99$ (1H, ddd, $J=2, J=4$, $J=6 \mathrm{~Hz}), 5.87(1 \mathrm{H}, \mathrm{br} \mathrm{s}), 5.20(1 \mathrm{H}, \mathrm{br} \mathrm{s}), 4.23(1 \mathrm{H}, \mathrm{t}, J=2 \mathrm{~Hz}), 3.98(1 \mathrm{H}, \mathrm{br} \mathrm{s}), 3.10(1 \mathrm{H}$, ddd, $J=2, J=4, J=6 \mathrm{~Hz}), 2.71(1 \mathrm{H}, \mathrm{ddd}, J=2, J=6, J=4 \mathrm{~Hz}) \cdot{ }^{13} \mathrm{C}-\mathrm{NMR}\left(125 \mathrm{MHz}, \mathrm{CDCl}_{3}\right), \delta$ : 175.7, 138.3, 132.4, 130.8, 129.2, 128.4, 128.4, 127.9, 127.4, 85.7, 63.0, 45.2. IR (neat): 3356, 2924, 1668, and $1456 \mathrm{~cm}^{-1}$. 